OCHA-PP-139

NDA-FP-??

August 1999

\title{
AXION-DILATONIC CONFORMAL ANOMALY FROM AdS/CFT CORRESPONDENCE
}

\author{
SHIN'ICHI NOJIRI円, SERGEI D. ODINTSOV ${ }^{\circledR}$, \\ SACHIKO OGUSHI ${ }^{\bigcirc}$, AKIO SUGAMOTO ${ }^{\complement}$ 田, \\ and Мiно YAMAMOTO? \\ Department of Mathematics and Physics \\ National Defence Academy, Hashirimizu Yokosuka 239, JAPAN \\ Tomsk Pedagogical University, 634041 Tomsk, RUSSIA \\ $\odot$ Department of Physics, Ochanomizu University \\ Otsuka, Bunkyou-ku Tokyo 112, JAPAN
}

\begin{abstract}
We discuss general multidimensional axion-dilatonic AdS gravity which may correspond to bosonic sector of Gibbons-Green-Perry (compactified) IIB supergravity with RR-scalar (axion). Using AdS/CFT correspondence the 4d conformal anomaly on axion-dilaton-gravitational background is found from SG side. It is shown that for IIB SG with axion such conformal anomaly coincides with the one obtained from QFT calculation in $\mathcal{N}=4$ super YangMills theory conformally coupled with $\mathcal{N}=4$ conformal SG. Brief discussion on possibility to apply these results for gauged SGs is also presented.
\end{abstract}

\footnotetext{
${ }^{1}$ nojiri@cc.nda.ac.jp

2 odintsov@mail.tomsknet.ru

3 JSPS Research Fellow, g9970503@edu.cc.ocha.ac.jp

${ }^{4}$ sugamoto@phys.ocha.ac.jp

5 g9840530@edu.cc.ocha.ac.jp
} 
One of the important tests of AdS/CFT correspondence (for an introduction and review, see [1]) is SG side calculation of conformal anomaly also called Weyl or trace anomaly (for a review, see [2]). This may also help in the construction of new examples of anomaly in the situation when perturbative (QFT) calculations are extremely complicated. The method to make such calculation (from SG side) has been suggested in ref. [3]. This evaluation has been tested for $\mathcal{N}=4$ super YM theory in refs. [4, 5, 6, 7, 8] and for $2 \mathrm{~d}$ superconformal theory [4, 9, mainly on pure gravitational background. Exact agreement with QFT conformal anomaly has been established in the leading order of large $N$ expansion. Moreover, it has been found agreement [10, 11] between AdS/CFT conformal anomaly and perturbative QFT anomaly in $\mathcal{N}=2 \mathrm{SCFT}$ even in the next to leading order term.

In ref. [4] the AdS/CFT correspondence on the level of trace anomaly has been successfully checked on dilaton-gravitational background. It has been demonstrated that boundary side is $\mathcal{N}=4$ super YM theory conformally coupled with $\mathcal{N}=4$ conformal SG. However, the important question remains: May other fields spoil AdS/CFT correspondence? To answer this question we consider 5 d dilaton-axionic AdS gravity. Our action is actually motivated by bosonic sector of SG model due to Gibbons, Green and Perry 12 which contains axion. This IIB SG represents the special case of our action. Other SGs maybe considered as well.

We found SG side conformal anomaly which exactly agrees with perturbative conformal anomaly in $\mathcal{N}=4$ super YM theory conformally coupled with $\mathcal{N}=4$ conformal supergravity. It completes the proof of AdS/CFT correspondence between two theories 14 . It is imporatant that background axion and dilaton are kept in the calculation of conformal anomaly.

We start with the following action:

$$
\begin{array}{r}
S=\frac{1}{16 \pi G}\left\{\int _ { M _ { d + 1 } } d ^ { d + 1 } x \sqrt { - \hat { G } } \left(\hat{R}+X(\phi, \chi)(\hat{\nabla} \phi)^{2}+Y(\phi, \chi) \hat{\Delta} \phi\right.\right. \\
\left.\left.+Z(\phi, \chi)(\hat{\nabla} \chi)^{2}+W(\phi, \chi) \hat{\Delta} \chi+4 \lambda^{2}\right)+\int_{M_{d}} d^{d} x \sqrt{-\hat{g}}\left(2 \hat{\nabla}_{\mu} n^{\mu}+\alpha\right)\right\} .
\end{array}
$$

Here $\phi$ and $\chi$ are dilaton and axion (or RR-scalar), respectively, and $M_{d+1}$ is the $d+1$ dimensional manifold whose boundary is the $d$ dimensional manifold $M_{d}$ and $n_{\mu}$ is the unit normal vector to $M_{d}$. We choose the metric $\hat{G}_{\mu \nu}$ on 
$M_{d+1}$ and the metric $\hat{g}_{\mu \nu}$ on $M_{d}$ in the following form

$$
d s^{2} \equiv \hat{G}_{\mu \nu} d x^{\mu} d x^{\nu}=\frac{l^{2}}{4} \rho^{-2} d \rho d \rho+\sum_{i=1}^{d} \hat{g}_{i j} d x^{i} d x^{j}, \quad \hat{g}_{i j}=\rho^{-1} g_{i j}
$$

Here $l$ is related with $\lambda^{2}$ by $4 \lambda^{2}=-d(d-1) / l^{2}$. Note that we follow to method of calculation in ref. [4] where dilatonic gravity has been considered.

We expand $\phi$ and $\chi$ as power series on $\rho$ :

$$
\begin{aligned}
\phi & =\phi_{(0)}+\rho \phi_{(1)}+\rho^{2} \phi_{(2)}+\cdots+\rho^{\frac{d}{2}} \phi_{(d / 2)}-\rho^{\frac{d}{2}} \ln \rho \psi+\mathcal{O}\left(\rho^{\frac{d}{2}+1}\right) \\
\chi & =\chi_{(0)}+\rho \chi_{(1)}+\rho^{2} \chi_{(2)}+\cdots+\rho^{\frac{d}{2}} \chi_{(d / 2)}-\rho^{\frac{d}{2}} \ln \rho \chi+\mathcal{O}\left(\rho^{\frac{d}{2}+1}\right) \\
g_{i j} & =g_{(0) i j}+\rho g_{(1) i j}+\rho^{2} g_{(2) i j}+\cdots+\rho^{\frac{d}{2}} g_{(d / 2) i j}-\rho^{\frac{d}{2}} \ln \rho h_{i j}+\mathcal{O}\left(\rho^{\frac{d}{2}+1}\right) .
\end{aligned}
$$

In the following, we abbreviate the index "(0)" if there is no any confusion and the action (1) diverges in general since the action contains the infinite volume integration on $M_{d+1}$. The action is regularized by introducing the infrared cutoff $\epsilon$ and replacing

$$
\int d^{d+1} x \rightarrow \int d^{d} x \int_{\epsilon} d \rho,\left.\quad \int_{M_{d}} d^{d} x(\cdots) \rightarrow \int d^{d} x(\cdots)\right|_{\rho=\epsilon} .
$$

The subtraction of the terms proportional to the inverse power of $\epsilon$ does not break the invariance under the scale transformation $\delta g_{\mu \nu}=2 \delta \sigma g_{\mu \nu}$ and $\delta \epsilon=2 \delta \sigma \epsilon$. When $d$ is even, however, the term proportional to $\ln \epsilon$ appears. This term is not invariant under the scale transformation and the subtraction of the $\ln \epsilon$ term breaks the invariance. The variation of the $\ln \epsilon$ term under the scale transformation is finite when $\epsilon \rightarrow 0$ and should be canceled by the variation of the finite term (which does not depend on $\epsilon$ ) in the action since the original action (11) is invariant under the scale transformation. Therefore the $\ln \epsilon$ term $S_{\ln }$ gives the Weyl anomaly $T$ of the action renormalized by the subtraction of the terms which diverge when $\epsilon \rightarrow 0$ by

$$
S_{\ln }=-\frac{1}{2} \int d^{4} x \sqrt{-g} T \text {. }
$$

Note that a bit different methods to calculate anomaly have been discussed in refs. [6, 8]. Nevertheless, the final result is unique. When $d=4, S_{\ln }$ is 
given by

$$
\begin{aligned}
S_{\mathrm{ln}} & =\frac{1}{16 \pi G} \int d^{4} x \sqrt{-g}\left\{\frac{1}{2 \ell^{i j}} g^{k l}\left(g_{(1) i j} g_{(1) k l}-g_{(1) i k} g_{(1) j l}\right)\right. \\
& +\frac{\ell}{2}\left(R^{i j}-\frac{1}{2} g^{i j} R\right) g_{(1) i j}-\frac{2}{\ell}\left\{\left(X-Y^{\prime}\right) \phi_{(1)} \phi_{(1)}+\left(Z-W^{*}\right) \chi_{(1)} \chi_{(1)}\right. \\
& \left.-\left(Y^{*}+W^{\prime}\right) \phi_{(1)} \chi_{(1)}\right\}-\frac{\ell}{2} g^{i j}\left\{\left(X^{\prime} \phi_{(1)}+X^{*} \chi_{(1)}\right) \partial_{i} \phi \partial_{j} \phi\right. \\
& \left.+\left(Z^{\prime} \phi_{(1)}+Z^{*} \chi_{(1)}\right) \partial_{i} \chi \partial_{j} \chi\right\}-\ell g^{i j}\left\{X \partial_{i} \phi \partial_{j} \phi_{(1)}+Z \partial_{i} \chi \partial_{j} \chi_{(1)}\right\} \\
& +\frac{\ell}{2} g^{i k} g^{j l} g_{(1) k l}\left\{X \partial_{i} \phi \partial_{j} \phi+Z \partial_{i} \chi \partial_{j} \chi\right\}-\frac{\ell}{4} g^{k l} g^{i j} g_{(1) k l}\left\{X \partial_{i} \phi \partial_{j} \phi+Z \partial_{i} \chi \partial_{j} \chi\right\} \\
& -\frac{\ell}{2}\left\{\left(Y^{\prime} \phi_{(1)}+Y^{*} \chi_{(1)}\right) \triangle \phi+\left(W^{\prime} \phi_{(1)}+W^{*} \chi_{(1)}\right) \triangle \chi+Y \triangle \phi_{(1)}+W \triangle \chi_{(1)}\right\} \\
& -\frac{\ell}{2}\left\{Y \frac{1}{\sqrt{-g}} \partial_{i}\left(\sqrt{-g}\left(\frac{1}{2} g^{k l} g_{(1) k l} g^{i j}-g^{i k} g_{(1) k l} g^{j l}\right) \partial_{j} \phi\right)\right. \\
& \left.+W \frac{1}{\sqrt{-g}} \partial_{i}\left(\sqrt{-g}\left(\frac{1}{2} g^{k l} g_{(1) k l} g^{i j}-g^{i k} g_{(1) k l} g^{j l}\right) \partial_{j} \chi\right)\right\} . \\
\triangle \cdot \equiv & \partial_{i}\left(\sqrt{-g} g^{i j} \partial_{j} \cdot\right)
\end{aligned}
$$

Here " " "and "* " express the derivative with respect to $\phi$ and $\chi$, respectively. We can solve the equations of motion given by the variation of $S_{\ln }$ with respect to $g_{(1) i j}, \phi_{(1)}, \chi_{(1)}$. The equation of motion for $g_{(1) i j}$ is

$$
\begin{aligned}
0= & \frac{1}{\ell}\left(g^{i j} g^{k l} g_{(1) k l}-g^{i k} g^{j l} g_{(1) k l}\right)+\frac{\ell}{2}\left(R^{i j}-\frac{1}{2} g^{i j} R\right) \\
& -\frac{\ell}{2} V\left(\frac{1}{2} g^{k l} g^{i j}-g^{i k} g^{j l}\right) \partial_{k} \phi \partial_{l} \phi-\frac{\ell}{2} K\left(\frac{1}{2} g^{k l} g^{i j}-g^{i k} g^{j l}\right) \partial_{k} \chi \partial_{l} \chi \\
& +\frac{\ell}{2} Q\left(\frac{1}{2} g^{k l} g^{i j}-g^{i k} g^{j l}\right) \partial_{k} \chi \partial_{l} \phi .
\end{aligned}
$$

Here $V, K, Q$ are defined as follows:

$$
V \equiv X-Y^{\prime}, \quad K \equiv Z-W^{*}, \quad Q \equiv W^{\prime}+Y^{*} .
$$

The equations of motion for $\phi_{(1)}$ and $\chi_{(1)}$ are

$$
0=-\frac{4}{\ell} V \phi_{(1)}+\frac{2}{\ell} Q \chi_{(1)}-\frac{\ell}{2} V^{\prime} g^{i j} \partial_{i} \phi \partial_{j} \phi
$$




$$
\begin{aligned}
& -\frac{\ell}{2} K^{\prime} g^{i j} \partial_{i} \chi \partial_{j} \chi+\frac{\ell}{2} Q^{\prime} g^{i j} \partial_{i} \phi \partial_{j} \chi \\
& +\frac{\ell}{\sqrt{-g}} \partial_{j}\left\{\sqrt{-g} V g^{i j} \partial_{i} \phi\right\}-\frac{\ell}{2 \sqrt{-g}} \partial_{j}\left\{\sqrt{-g} Q g^{i j} \partial_{i} \chi\right\} \\
0= & -\frac{4}{\ell} K \chi_{(1)}+\frac{2}{\ell} Q \phi(1)-\frac{\ell}{2} K^{*} g^{i j} \partial_{i} \chi \partial_{j} \chi \\
& -\frac{\ell}{2} V^{*} g^{i j} \partial_{i} \phi \partial_{j} \phi+\frac{\ell}{2} Q^{*} g^{i j} \partial_{i} \phi \partial_{j} \chi \\
& +\frac{\ell}{\sqrt{-g}} \partial_{j}\left\{\sqrt{-g} K g^{i j} \partial_{i} \chi\right\}-\frac{\ell}{2 \sqrt{-g}} \partial_{j}\left\{\sqrt{-g} Q g^{i j} \partial_{i} \phi\right\} .
\end{aligned}
$$

Then $g_{(1) i j}, \phi_{(1)}, \chi_{(1)}$ can be given in terms of $g_{i j}, \phi, \chi$.

$$
\begin{aligned}
g_{(1) i j} & =\frac{\ell^{2}}{2}\left(R_{i j}-\frac{1}{6} g_{i j} R\right)+\frac{\ell^{2}}{2} V\left\{\partial_{i} \phi \partial_{j} \phi-\frac{1}{6} g_{i j} g^{k l} \partial_{k} \phi \partial_{l} \phi\right\} \\
& +\frac{\ell^{2}}{2} K\left\{\partial_{i} \chi \partial_{j} \chi-\frac{1}{6} g_{i j} g^{k l} \partial_{k} \chi \partial_{l} \chi\right\}-\frac{\ell^{2}}{2} Q\left\{\partial_{i} \phi \partial_{j} \chi-\frac{1}{6} g_{i j} g^{k l} \partial_{k} \phi \partial_{l} \chi\right\} \\
\phi_{(1)} & =\left\{-\frac{\ell^{2}}{16}\left(\frac{Q \xi}{K V}-2 \frac{V^{\prime}}{V}\right) g^{i j} \partial_{i} \phi \partial_{j} \phi-\frac{\ell^{2}}{16}\left(2 \frac{\eta}{V}-\frac{Q K^{*}}{K V}\right) g^{i j} \partial_{i} \chi \partial_{j} \chi\right. \\
& \left.+\frac{\ell^{2}}{8}\left(\frac{K^{\prime} Q}{K V}+2 \frac{V^{*}}{V}\right) g^{i j} \partial_{i} \phi \partial_{j} \chi\right\}\left(1-\frac{1}{4} \frac{Q^{2}}{K V}\right)^{-1}+\frac{\ell^{2}}{4} \Delta \phi \\
\chi_{(1)} & =\left\{-\frac{\ell^{2}}{16}\left(2 \frac{\xi}{K}-\frac{Q V^{\prime}}{K V}\right) g^{i j} \partial_{i} \phi \partial_{j} \phi-\frac{\ell^{2}}{16}\left(\frac{Q \eta}{K V}-2 \frac{K^{*}}{K}\right) g^{i j} \partial_{i} \chi \partial_{j} \chi\right. \\
& \left.+\frac{\ell^{2}}{8}\left(\frac{V^{*} Q}{K V}+2 \frac{K^{\prime}}{K}\right) g^{i j} \partial_{i} \phi \partial_{j} \chi\right\}\left(1-\frac{1}{4} \frac{Q^{2}}{K V}\right)^{-1}+\frac{\ell^{2}}{4} \triangle \chi
\end{aligned}
$$

Here $\eta, \xi$ are defined as follows:

$$
\eta \equiv K^{\prime}+Q^{*}=Z^{\prime}+Y^{* *}, \quad \xi \equiv V^{*}+Q^{\prime}=X^{*}+W^{\prime \prime} .
$$

For substituing (11), (12), (13) into the action (6), we rewrite (6) as follows

$$
\begin{aligned}
S_{\ln }= & \frac{1}{16 \pi G} \int d^{4} x \sqrt{-g}\left\{\frac{1}{2 \ell} g^{i j} g^{k l}\left(g_{(1) i j} g_{(1) k l}-g_{(1) i k} g_{(1) j l}\right)\right. \\
& +\frac{\ell}{2}\left(R^{i j}-\frac{1}{2} g^{i j} R\right) g_{(1) i j}-\frac{2}{\ell}\left\{V \phi_{(1)} \phi_{(1)}+K \chi_{(1)} \chi_{(1)}-Q \phi_{(1)} \chi_{(1)}\right\} \\
& -\frac{\ell}{2} V\left(\frac{1}{2} g^{k l} g^{i j}-g^{i k} g^{j l}\right) g_{(1) i j} \partial_{k} \phi \partial_{l} \phi-\frac{\ell}{2} K\left(\frac{1}{2} g^{k l} g^{i j}-g^{i k} g^{j l}\right) g_{(1) i j} \partial_{k} \chi \partial_{l} \chi
\end{aligned}
$$




$$
\begin{aligned}
& +\frac{\ell}{2} Q\left(\frac{1}{2} g^{k l} g^{i j}-g^{i k} g^{j l}\right) g_{(1) i j} \partial_{k} \phi \partial_{l} \chi-\frac{\ell}{2}\left(V^{\prime} \phi_{(1)}+V^{*} \chi_{(1)}\right) g^{i j} \partial_{i} \phi \partial_{j} \phi \\
& -\frac{\ell}{2}\left(K^{*} \chi_{(1)}+K^{\prime} \phi_{(1)}\right) g^{i j} \partial_{i} \chi \partial_{j} \chi+\frac{\ell}{2}\left(Q^{\prime} \phi_{(1)}+Q^{*} \chi_{(1)}\right) g^{i j} \partial_{i} \phi \partial_{j} \chi \\
& \left.-\ell V g^{i j} \partial_{i} \phi \partial_{j} \phi_{(1)}-\ell K g^{i j} \partial_{i} \chi \partial_{j} \chi_{(1)}-\frac{\ell}{2} Q\left(g^{i j} \partial_{i} \phi_{(1)} \partial_{j} \chi+g^{i j} \partial_{i} \phi \partial_{j} \chi\right)\right\}
\end{aligned}
$$

Substituing (11), (12), (13) into this action, we find

$$
\begin{aligned}
S_{\mathrm{ln}} & =\frac{\ell^{3}}{16 \pi G} \int d^{4} x \sqrt{-g}\left\{\frac{1}{8} R^{i j} R_{i j}-\frac{1}{24} R^{2}\right. \\
& +\frac{V}{4}\left(R^{i j} \partial_{i} \phi \partial_{j} \phi-\frac{1}{3} R g^{i j} \partial_{i} \phi \partial_{j} \phi\right)+\frac{K}{4}\left(R^{i j} \partial_{i} \chi \partial_{j} \chi-\frac{1}{3} R g^{i j} \partial_{i} \chi \partial_{j} \chi\right) \\
& -\frac{Q}{4}\left(R^{i j} \partial_{i} \phi \partial_{j} \chi-\frac{1}{3} R g^{i j} \partial_{i} \phi \partial_{j} \chi\right)-\frac{1}{12}\left\{\left(V g^{i j} \partial_{i} \phi \partial_{j} \phi\right)^{2}+\left(K g^{i j} \partial_{i} \chi \partial_{j} \chi\right)^{2}\right. \\
& \left.+\left(Q g^{i j} \partial_{i} \phi \partial_{j} \chi\right)^{2}\right\}-\frac{K V}{4}\left\{\frac{1}{3}\left(g^{i j} \partial_{i} \phi \partial_{j} \phi\right)\left(g^{k l} \partial_{k} \chi \partial_{l} \chi\right)-\left(g^{i j} \partial_{i} \phi \partial_{j} \chi\right)^{2}\right\} \\
& -\frac{V Q}{6}\left(g^{i j} \partial_{i} \phi \partial_{j} \phi\right)\left(g^{k l} \partial_{k} \phi \partial_{l} \chi\right)-\frac{K Q}{6}\left(g^{i j} \partial_{i} \chi \partial_{j} \chi\right)\left(g^{k l} \partial_{k} \chi \partial_{l} \phi\right) \\
& +\left[\frac{1}{32}\left(\frac{V^{\prime 2}}{V}+\frac{\xi^{2}}{K}-\frac{V^{\prime} Q \xi}{V K}\right)\left(g^{i j} \partial_{i} \phi \partial_{j} \phi\right)^{2}\right. \\
& +\frac{1}{32}\left(\frac{K^{* 2}}{K}+\frac{\eta^{2}}{V}-\frac{K^{*} Q \eta}{V K}\right)\left(g^{i j} \partial_{i} \chi \partial_{j} \chi\right)^{2} \\
& +\frac{1}{8}\left(\frac{K^{\prime 2}}{K}+\frac{V^{* 2}}{V}-\frac{V^{*} Q K^{\prime}}{V K}\right)\left(g^{i j} \partial_{i} \phi \partial_{j} \chi\right)^{2} \\
& +\frac{1}{16}\left(-\frac{K^{*}}{K} \xi-\frac{V^{\prime}}{V} \eta+\frac{Q}{2 V K}\left(K^{*} V^{\prime}+\xi \eta\right)\right)\left(g^{i j} \partial_{i} \phi \partial_{j} \phi\right)\left(g^{k l} \partial_{k} \chi \partial_{l} \chi\right) \\
& +\frac{1}{16}\left(-2 \frac{K^{\prime}}{K} \xi+2 \frac{V^{\prime} V^{*}}{V} \eta+\frac{Q}{V K}\left(K^{\prime} V^{\prime}-V^{*} \xi\right)\right)\left(g^{i j} \partial_{i} \phi \partial_{j} \phi\right)\left(g^{k l} \partial_{k} \phi \partial_{l} \chi\right) \\
& \left.+\frac{1}{16}\left(-2 \frac{V^{*}}{V} \eta+2 \frac{K^{\prime} K^{*}}{K} \eta+\frac{Q}{V K}\left(K^{*} V^{*}-K^{\prime} \eta\right)\right)\left(g^{i j} \partial_{i} \phi \partial_{j} \chi\right)\left(g^{k l} \partial_{k} \chi \partial_{l} \chi\right)\right] \\
& \times\left(1-\frac{Q^{2}}{4 K V}\right)^{-1}+\frac{V}{8}(\triangle \phi)^{2}+\frac{K}{8}(\triangle \chi)^{2}-\frac{Q}{4} \triangle \phi \triangle \chi \\
& -\frac{1}{16}\left[\left(\frac{Q}{K} \xi-2 V^{\prime}\right) g^{k l} \partial_{k} \phi \partial_{l} \phi \triangle \phi+\left(\frac{Q}{V} \eta-2 K^{*}\right) g^{k l} \partial_{k} \chi \partial_{l} \chi \triangle \chi\right.
\end{aligned}
$$




$$
\begin{aligned}
& -\frac{Q}{2 V}\left(\frac{Q}{K} \xi-2 V^{\prime}\right) g^{k l} \partial_{k} \phi \partial_{l} \phi \triangle \chi \\
& \left.-\frac{Q}{2 K}\left(\frac{Q}{V} \eta-2 K^{*}\right) g^{k l} \partial_{k} \chi \partial_{l} \chi \triangle \phi\right] \times\left(1-\frac{Q^{2}}{4 K V}\right)^{-1} \\
& \left.+\frac{V^{*}}{4} g^{k l} \partial_{k} \phi \partial_{l} \chi \frac{1}{\sqrt{-g}} \partial_{j}\left(\sqrt{-g} g^{i j} \partial_{i} \phi\right)+\frac{K^{\prime}}{4} g^{k l} \partial_{k} \phi \partial_{l} \chi \triangle \chi\right\}
\end{aligned}
$$

If we define

$$
\left(\begin{array}{c}
X^{1} \\
X^{2}
\end{array}\right) \equiv\left(\begin{array}{c}
\phi \\
\chi
\end{array}\right), \quad h_{\mu \nu} \equiv\left(\begin{array}{cc}
V & -\frac{Q}{2} \\
-\frac{Q}{2} & K
\end{array}\right)
$$

we obtain

$$
h \equiv \operatorname{det} h_{\mu \nu}=V K-\frac{Q^{2}}{4}, \quad h^{\mu \nu}=\frac{1}{h}\left(\begin{array}{cc}
K & \frac{Q}{2} \\
\frac{Q}{2} & V
\end{array}\right) .
$$

Then Eqs.(11), (12) and (13) can be rewritten as follows:

$$
\begin{aligned}
g_{(1) i j} & =\frac{l^{2}}{2}\left\{\left(R_{i j}-\frac{1}{6} g_{i j} R\right)+h_{\mu \nu}\left(\partial_{i} X^{\mu} \partial_{j} X^{\nu}-\frac{1}{6} g_{i j}\left(\partial X^{\mu} \cdot \partial X^{\nu}\right)\right)\right\} \\
X_{(1)}^{\mu} & =\frac{l^{2}}{4} \Gamma_{\rho \sigma}^{\mu}\left(\partial X^{\rho} \cdot \partial X^{\sigma}\right)+\frac{l^{2}}{4} \triangle X^{\mu} .
\end{aligned}
$$

Here

$$
\left(\partial X^{\mu} \cdot \partial X^{\nu}\right) \equiv g^{i j} \partial_{i} X^{\mu} \partial_{j} X^{\nu}
$$

and $\Gamma_{\nu \rho}^{\mu}$ is a connection on the target manifold:

$$
\Gamma_{\nu \rho}^{\mu}=\frac{1}{2} h^{\mu \tau}\left(h_{\nu \tau, \rho}+h_{\rho \tau, \nu}-h_{\nu \rho, \tau}\right) .
$$

On the other hand, $S_{\ln }$ can be rewritten as follows

$$
\begin{aligned}
S_{\mathrm{ln}} & =\frac{1}{16 \pi G} \int d^{4} x \sqrt{-g}\left\{\frac{1}{2 l} g^{i j} g^{k l}\left(g_{(1) i j} g_{(1) k l}-g_{(1) i k} g_{(1) j l}\right)\right. \\
& +\frac{l}{2}\left(R^{i j}-\frac{1}{2} R g^{i j}\right) g_{(1) i j}-\frac{2}{l} h_{\mu \nu} X_{(1)}^{\mu} X_{(1)}^{\nu} \\
& -\frac{l}{2} h_{\mu \nu}\left(\frac{1}{2} g^{k l} g^{i j}-g^{i k} g^{j l}\right) g_{(1) i j} \partial_{k} X^{\mu} \partial_{l} X^{\nu} \\
& \left.-\frac{l}{2} h_{\mu \nu, \rho} X_{(1)}^{\rho} g^{i j} \partial_{i} X^{\mu} \partial_{j} X^{\nu}-l h_{\mu \nu} g^{i j} \partial_{i} X^{\mu} \partial_{j} X_{(1)}^{\nu}\right\}
\end{aligned}
$$


Substituting (18) and (18) into (21) and integrating by parts, we obtain

$$
\begin{aligned}
S_{\ln } & =\frac{1}{16 \pi G} \int d^{4} x \sqrt{-g}\left[l^{3}\left(-\frac{1}{24} R^{2}+\frac{1}{8} R_{i j} R^{i j}\right)\right. \\
& +\frac{l^{3}}{4} R^{i j} h_{\mu \nu} \partial_{i} X^{\mu} \partial_{j} X^{\nu}-\frac{l^{3}}{12} R h_{\mu \nu}\left(\partial X^{\mu} \cdot \partial X^{\nu}\right) \\
& -\frac{l^{3}}{24}\left\{h_{\mu \nu}\left(\partial X^{\mu} \cdot \partial X^{\nu}\right)\right\}^{2}+\frac{l^{3}}{8} h_{\mu \nu} h_{\rho \sigma}\left(\partial X^{\mu} \cdot \partial X^{\rho}\right)\left(\partial X^{\nu} \cdot \partial X^{\sigma}\right) \\
& \left.+\frac{l^{8}}{8} h_{\mu \nu}\left\{\triangle X^{\mu}+\Gamma_{\rho \sigma}^{\mu}\left(\partial X^{\rho} \cdot \partial X^{\sigma}\right)\right\}\left\{\triangle X^{\nu}+\Gamma_{\tau \eta}^{\nu}\left(\partial X^{\tau} \cdot \partial X^{\eta}\right)\right\}\right] .
\end{aligned}
$$

If we put

$$
h_{\mu \nu}=\left(\begin{array}{ll}
2 & 0 \\
0 & c
\end{array}\right),(c \text { is an arbitrary constant }), \quad X^{2}=0
$$

the previous result in [4] for dilatonic gravity can be reproduced.

If we choose the action as motivated by the bosonic sector of type IIB supergravity with RR-scalar 12

$$
S=\frac{1}{16 \pi G} \int_{M_{d+1}} d^{d+1} x \sqrt{-\hat{G}}\left(\hat{R}+\frac{1}{2}(\hat{\nabla} \phi)^{2}+\frac{1}{2} \mathrm{e}^{2 \phi}(\hat{\nabla} \chi)^{2}\right) .
$$

the functions $X(\phi, \chi), Y(\phi, \chi), Z(\phi, \chi), W(\phi, \chi)$ become

$$
X(\phi, \chi)=+\frac{1}{2}, \quad Z(\phi, \chi)=\frac{1}{2} \mathrm{e}^{2 \phi}, \quad Y(\phi, \chi)=W(\phi, \chi)=0 .
$$

Then the $\ln \epsilon$ terms of the action, corresponding to the anomaly, are written in following form.

$$
\begin{aligned}
S_{\mathrm{ln}} & =\frac{\ell^{3}}{16 \pi G} \int d^{4} x \sqrt{-g}\left\{\frac{1}{8} R^{i j} R_{i j}-\frac{1}{24} R^{2}\right. \\
& -\frac{1}{8}\left(R^{i j} \partial_{i} \phi \partial_{j} \phi-\frac{1}{3} R g^{i j} \partial_{i} \phi \partial_{j} \phi\right)+\frac{1}{8} \mathrm{e}^{2 \phi}\left(R^{i j} \partial_{i} \chi \partial_{j} \chi-\frac{1}{3} R g^{i j} \partial_{i} \chi \partial_{j} \chi\right) \\
& -\frac{1}{48}\left\{\left(g^{i j} \partial_{i} \phi \partial_{j} \phi\right)^{2}+\mathrm{e}^{4 \phi}\left(g^{i j} \partial_{i} \chi \partial_{j} \chi\right)^{2}\right\} \\
& +\frac{1}{16} \mathrm{e}^{2 \phi}\left\{\frac{1}{3}\left(g^{i j} \partial_{i} \phi \partial_{j} \phi\right)\left(g^{k l} \partial_{k} \chi \partial_{l} \chi\right)-\left(g^{i j} \partial_{i} \phi \partial_{j} \chi\right)^{2}\right\} \\
& -\frac{1}{16} \mathrm{e}^{4 \phi}\left(g^{i j} \partial_{i} \chi \partial_{j} \chi\right)^{2}+\frac{1}{4} \mathrm{e}^{2 \phi}\left(g^{i j} \partial_{i} \phi \partial_{j} \chi\right)^{2} \\
& \left.-\frac{1}{16}(\triangle \phi)^{2}+\frac{1}{16} \mathrm{e}^{2 \phi}(\triangle \chi)^{2}+\frac{1}{4} \mathrm{e}^{2 \phi} g^{k l} \partial_{k} \phi \partial_{l} \chi \triangle \chi\right\}
\end{aligned}
$$


The Weyl anomaly coming from the multiplets of $\mathcal{N}=4$ supersymmetric $U(N)$ or $S U(N)$ Yang-Mills conformally coupled with $\mathcal{N}=4$ conformal supergravity was calculated in 13$]:$ ?

$$
\begin{aligned}
T= & -\frac{N^{2}}{4(4 \pi)^{2}}\left[2\left(R_{i j} R^{i j}-\frac{1}{3} R^{2}\right)+F^{i j} F_{i j}\right. \\
& \left.+4\left\{2\left(R^{i j}-\frac{1}{3} R g^{i j}\right) \partial_{i} \varphi^{*} \partial_{j} \varphi+|\triangle \varphi|^{2}\right\}+\cdots\right] .
\end{aligned}
$$

Here $F_{i j}$ is the field strength of $\mathrm{SU}(4)$ gauge fields, $\varphi$ is a complex scalar field which is a combination of dilaton and RR scalar and "..." expresses the terms containing other fields in $\mathcal{N}=4$ conformal supergravity multiplet and higher powers of the fields. It is also interesting that above conformal anomaly actually gives the (bosonic sector) of $\mathcal{N}=4$ conformal SG.

If we choose

$$
\frac{l^{3}}{16 \pi G}=\frac{2 N^{2}}{(4 \pi)^{2}}, \quad \varphi=\phi+\mathrm{e}^{\phi} \chi, \quad \varphi^{*}=-\phi+\mathrm{e}^{\phi} \chi
$$

and consider the background where only gravity and the scalar field $\varphi$ in the $\mathcal{N}=4$ conformal supergravity multiplet are non-trivial and other fields vanish in (27), Eq.(26) exactly reproduces the result in (27).

Thus, we got axion-dilatonic Weyl anomaly from SG side. It is remarkable that it coincides with perturbative result even in presence of axion, not only in dilaton-gravitational background.

It is not difficult to get the corresponding result in other dimensions (we discussed only $\mathrm{d} 4$ case). It would be of interest also to extend our results for gauged SGs. To do this one should add the axion-dilatonic (or in minimal case, only dilatonic) potential to initial action. This may help in construction of new versions of multidimensional gauged SGs. In its own turn, the analysis like above may also be useful in the extending of AdS/CFT correspondence to gauged SGs.

\section{References}

[1] O. Aharony, S. Gubser, J. Maldacena, H. Ooguri and Y. Oz, hepth/9905111.

\footnotetext{
${ }^{6}$ See Eqs. $(2.5)$ and $(2.6)$ in $[13]$.
} 
[2] M. Duff, Class.Quant.Grav. 11 (1994) 1387.

[3] E. Witten, Adv.Theor.Math.Phys. 2 (1998) 253.

[4] S. Nojiri and S.D. Odintsov, Phys.Lett. B444 (1998) 92, hepth/9810008.

[5] V. Balasubramanian and P. Kraus, hep-th/9902121.

[6] W. Mueck and K.S. Viswanathan, hep-th/9905048;

[7] M. Henningson and K. Skenderis, JHEP 9807023 (1998), hepth/9806087.

[8] P. Mansfield and D. Nolland, hep-th/9906054.

[9] M. Nishimura and Y. Tanii, hep-th/9904010.

[10] M. Blau, K.S. Narain and E. Gava, hep-th/9904179.

[11] S. Nojiri and S.D. Odintsov, hep-th/9903033.

[12] G.W. Gibbons, M.B. Green and M.J. Perry, Phys.Lett. B370 (1996) 37.

[13] H. Liu and A.A. Tseytlin, Nucl.Phys. B533 (1998) 88, preprint hepth/9804083. 